\title{
The Augmented Reality for Teaching Thai Students about the Human Heart
}

\author{
https://doi.org/10.3991/ijet.v13i06.8506 \\ Sumitra Nuanmeesri \\ Suan Sunandha Rajabhat University, Bangkok, Thailand \\ sumitra.nuessru.ac.th
}

\begin{abstract}
Biology is a science about living organisms. Organisms have complex systems consisting of complex organs. Focusing on the human body, if the organ or its structure is visually presented, the learners are more likely to understand it and its function. This research aims to explore the bilingual (Thai and English language), development of an augmented reality tool for use in teaching students about the human heart. The augmented reality application was evaluated by five experts, who analyzed its content consistency by using the Index of Item Objective Congruence (IOC), Diffusion of Innovation (DOI), and the content validity index (CVI), indicating that the augmented reality can be used for publicizing. A sample of 30 subjects were evaluated after AR training. It was determined that the learning result post AR obtained higher ratings when compared with the ratings prior to the use of augmented reality tool. The before and after augmented reality learning results were analyzed for statistical significance at $p$ value $<0.001$ with the use of a T-Test. Afterwards, the effectiveness of the tool was evaluated by users focusing on the acceptance of the augmented reality tool to teach the anatomy of the heart; the evaluation of which was based on the theory of Unified Theory Acceptance and Use of Technology (UTAUT) in which the results of the arithmetic mean and the standard deviation were 4.65 and 0.48 , respectively. This demonstrated that the users generally accepted the augmented reality tool to teach about the heart at the highest level.
\end{abstract}

Keywords-Augmented Reality, Human Heart, Teaching, Biology

\section{Introduction}

The educational management or the lesson planning for students in this era rely on modern tools instructors to attract the attention of the learners and give the best knowledge and learning experience to the learners. With the help of modern technology, the production of media must consider the major objective of using media which is to develop the learners' proficiency in all aspects [1]). The learners tend to understand the content via memorization of pictures and gain visual perception and real understanding. This means less of a burden on the teachers while reducing the difference between the learners [2]. 
Biology is a science that studies about living. The study of morphology, physiology, growth, behavior, evolution, ecology, and so on, concerning the living organisms at the cellular and organic levels, contain a wide range of content and detail which can lead the learners to have a negative attitude towards the subject. Moreover, the teachers might use media that does not promote learning. That tends to affect their understanding about biological principles. There is a lot of knowledge content, but the fundamental principle of biology (which is the closest to us) is the human body, and in particular the 'heart'. Humans are multicellular living organisms. It consists of multiple cells in the form of tissues. The organs and their systems are complex. Furthermore, the heart is an important organ which plays a huge role in continuously circulating blood to other organs in the body. The human heart is located in the chest span and slightly to the left. It is divided into four chambers: two on the top and the other two at the bottom. If there is some abnormality happening within the heart, it can be fatal. Heart disease is one of the main causes of death among Thai people as the average death rate is one person in seven, with the trend increasing annually. Due to its importance, the heart as well as its structural characteristics and functions should be well studied for better understanding about its function in order to protect and maintain it. In biology class, focusing on the human body, if the organ or its structure is visually presented, the learners tend to understand it and its function better [3]. However, for the internal organs, it is difficult to see their shapes, sizes, structures, and functions; even though there are figures and descriptions in textbooks, related documents or on the internet, as most of them are presented in the form of two dimensional images in which their characteristics or functions cannot be clearly seen [4]. In other words, this potentially affects the proficiency of the learners. In fact, the appropriate form of instructional media is the ability to effectively enhance learners' perception towards the use of computers. Computer Software should be developed to promote higher efficiency, facilitate the data processing system, and effectively present information, pictures, sound, and messages. For example, the virtual technology (Augmented Reality) is one of the innovations that can be applied to the area of education to stimulate, support, and promote learning experiences [5]. Barsom et al. did a systematic review on the effectiveness of augmented reality applications in medical training. They reported that augmented reality applications support blended learning in medical training and have gained public and scientific interest. In order to be of value, applications must be able to transfer information to the user. While promising, supporting evidence to date is lacking [6]. Wee et al. presented augmented and virtual reality in surgery the digital surgical environment including applications, limitations and legal pitfalls. A head mounted display (HMD) with either VR or AR (virtual or augmented reality respectively), will have great potential in the field of surgery. Their functionality has the potential for benefit in a range of clinical settings across the MDT and in medical education. First generation devices like GG have given us a glimpse of what AR can provide and despite its demise our appetite for new head mounted devices has not diminished [7]. New innovations like the Microsoft HoloLens and the emerging mass market of VR headsets would indicate that these technologies will become familiar to surgeons and inevitability we will find a way to integrate them into our day to day practice. The challenge of identifying compelling 
and valuable experiences for these modalities now begins along with validation of their benefits in all aspects of surgical care. The digital surgical environment is about to drastically change. Joyce reported that augmented reality magnetically tracked scalpels reduce tool switching though limitations in today's augmented reality technology fall short of creating an ideal immersive workflow by requiring the use of a monitor [8]. While this technology catches up, we recommend focusing efforts on enabling the easy creation of layered, complex reports, linking, and viewing information across systems. Reflecting upon our results, we argue for digitalization to focus not only on how to record increasing amounts of data but also how these data can be accessed in a more thoughtful way that draws upon the expertise and creativity of pathology professionals using the systems. There are also many companies that have developed augmented reality to teach students about the human body. There are no research studies on the development of augmented reality as a tool to teach the human body using Thai language.

The research had developed the augmented reality tool to teach the human body, especially the functioning of the heart in a bilingual setting (Thai and English language), with the use of the Android operational system which is available on smartphones and tablets. It can assist the students and interested people in studying about the human body with visual aids and without limitations in location. The tool can enable the students to gain more knowledge and understanding, and ultimately increase their interest in the subject. This study also aims to increase the learning potential for learners to catch up with the digital era. In addition, it can reduce the long term expenses and contribute to the learning society that stimulates, supports, and promotes education through media. It may also further expand the proficiency of the learners.

\section{$2 \quad$ Methodology}

The research methods for studying the development of the augmented reality tool to teach students about the heart, include the following steps:

\subsection{Documentary Research}

The analysis and data collection on human heart function was performed by the research by reviewing related theories and previous studies on human heart function to utilize data in developing the augmented reality tool. The human heart is comprised of four chambers $[9,16]:$ 1) The chamber on the top right (Right Atrium) 2) The chamber at the bottom right (Right Ventricle) 3) The chamber on the top left (Left Atrium) 4) The chamber at the bottom left (Left Ventricle). 


\subsection{The Design of the Augmented Reality for Teaching Thai Students about the Human Heart}

Based on collected information about human heart function, the heart components, heartbeat, and the blood circulation, the augmented reality model was constructed and installed in the system. The subjects were able to use their smartphones or tablets with the scanning application to scan the images used for enhancing the memorization process on heart structure. After scanning, the heart structure showed up in the form of three dimensional pictures.

\subsection{The Development of the Augmented Reality for Teaching Thai Students about the Human Heart}

Based on the design of the augmented reality tool for teaching about the heart, the information about the heart components, the heartbeat, and the blood circulation in each chamber were used for constructing the model of the augmented reality tool to teach heart anatomy. Subsequently, there was a construction of the contents promoting memorization skills by linking pictures together into the model of a heart. The users could use the augmented reality via smartphones or tablets with a scanning application to scan the heart pictures for virtual memorization of the structure, the heartbeat in each chamber, the blood circulation, and other functions of each chamber. The subjects had a choice in displaying the Thai or English language. Augmented Reality displays the blood flow with each heartbeat in each chamber as shown Figure 1 to Figure 3.

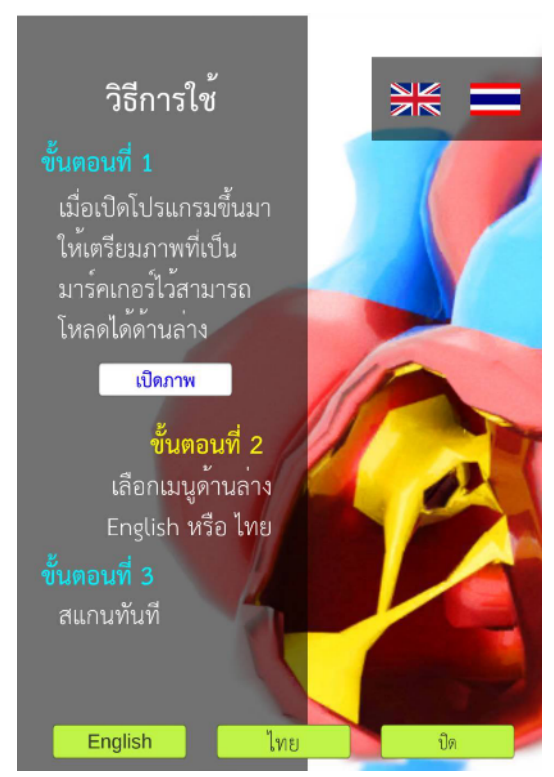

Fig. 1. Interface of the augmented reality for teaching Thai students about the human heart 


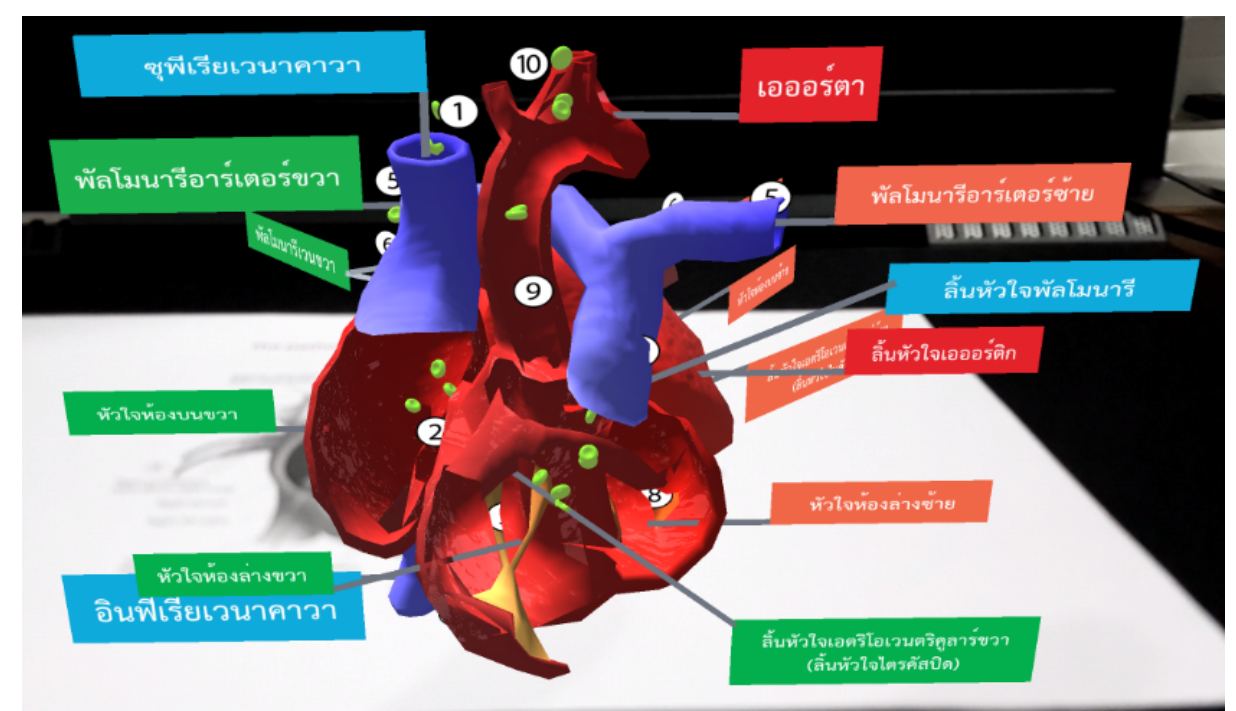

Fig. 2. Display Thai language in the augmented reality for teaching Thai students about the human heart

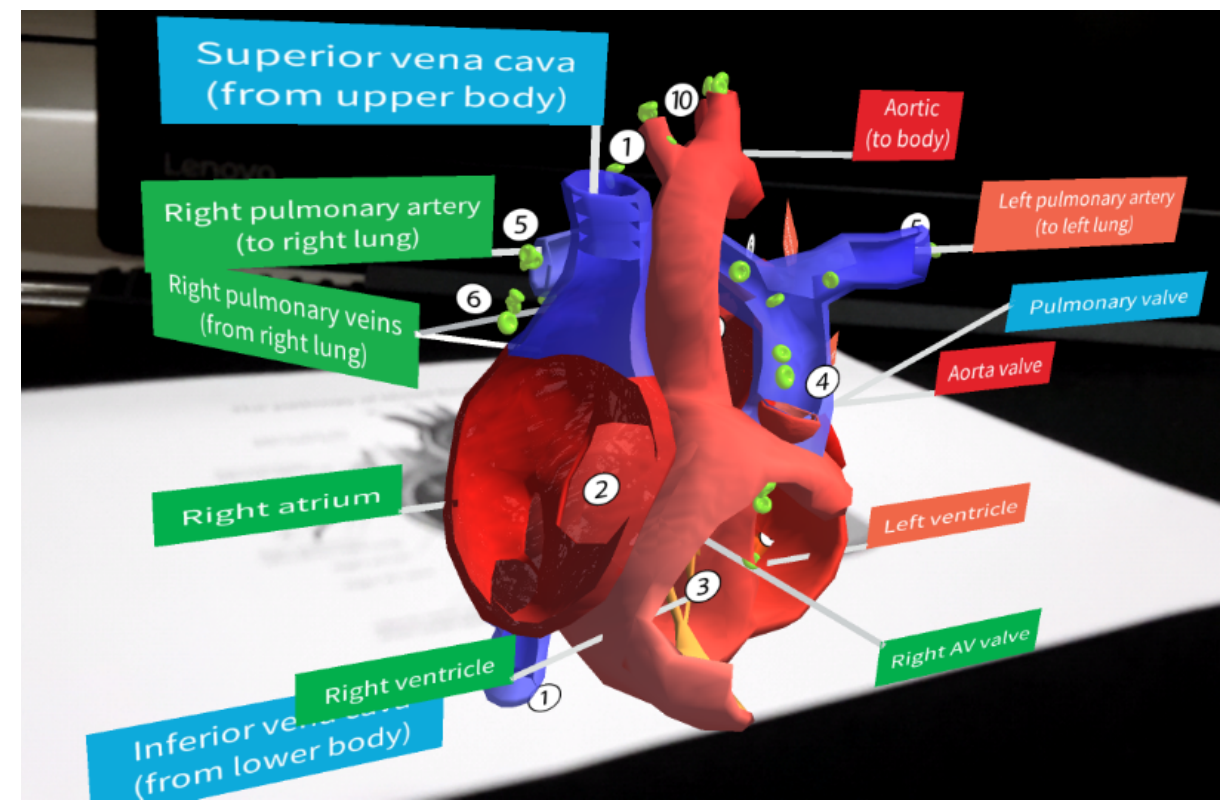

Fig. 3. Display English language in the augmented reality for teaching Thai students about the human heart 


\subsection{Evaluation of the Effectiveness of the Augmented Reality for Teaching Thai Students about the Human Heart}

The effectiveness of the augmented reality was evaluated by five experts who were specialized in the field of information technology and biology. The evaluation used the analysis of content consistency (Index of Item Objective Congruence: IOC) [10] by which the experts to provide scores. If the criteria determined by the IOC value of each indicator was higher than 0.5 (the highest IOC value is 1 ), it means the augmented reality tool met the objective, possessed content suitable for education purposes, and that the augmented reality tool worked effectively. The IOC value of each indicator was 0.8 , indicating that the developed augmented reality tool met the objective and in content consistency and is suitable for use in education about the heart.

Subsequently, the experts also used the Diffusion of Innovation theory (DOI) to evaluate five parameters [11]), being 1) more advantageous or have better performance 2) usability, 3) noticeability, 4) consistency with the demand or experiences of the learners who would potentially adopt the innovation, and 5) the result of which could be shown in advance [12]. It is necessary to see if the innovation could be adopted by the sample group. The data was analyzed to find the mean value and the standard deviation value in order to assess the innovation dissemination of the augmented reality tool to teach about the heart. The evaluation results of the DOI in all five aspects evaluated by the experts were 1) the arithmetic mean and the standard deviation of "more advantages or better performance" were 4.40 and 0.55 , respectively. It showed that this criterion was rated at a high level. 2) The arithmetic mean and the standard deviation of "usability" were 4.40 and 0.55 , respectively. It showed that this criterion was also rated at a high level. 3) The arithmetic mean and the standard deviation of "noticeability" were 4.40 and 0.55 , respectively. It showed that this criterion was rated at a high level. 4) The arithmetic mean and the standard deviation of "the consistency with the demand or experience of the subjects who would potentially adopt the innovation" were 4.20 and 0.45 , respectively. It showed that this criterion was rated at a high level. 5) The arithmetic mean and the standard deviation of "the result which could be shown in advance" were 4.40 and 0.55 , respectively. It showed that this criterion was rated at a high level. The arithmetic mean and the standard deviation of the overall image of the innovation dissemination were 4.36 and 0.49 , respectively. It showed that the augmented reality tool to teach about the heart was accepted to be used at a high level.

\subsection{The Dissemination of the Augmented Reality for Teaching Thai Students about the Human Heart}

The researcher tested the augmented reality to teach 30 subjects about the heart. The subjects were students at elementary level. Permission to conduct the study was obtained prior to the study the sample group was randomly selected from volunteers. Training was provided via a lecture and workshop demonstrating how to use the augmented reality tool to teach about heart function [13] as shown Figure 4. 


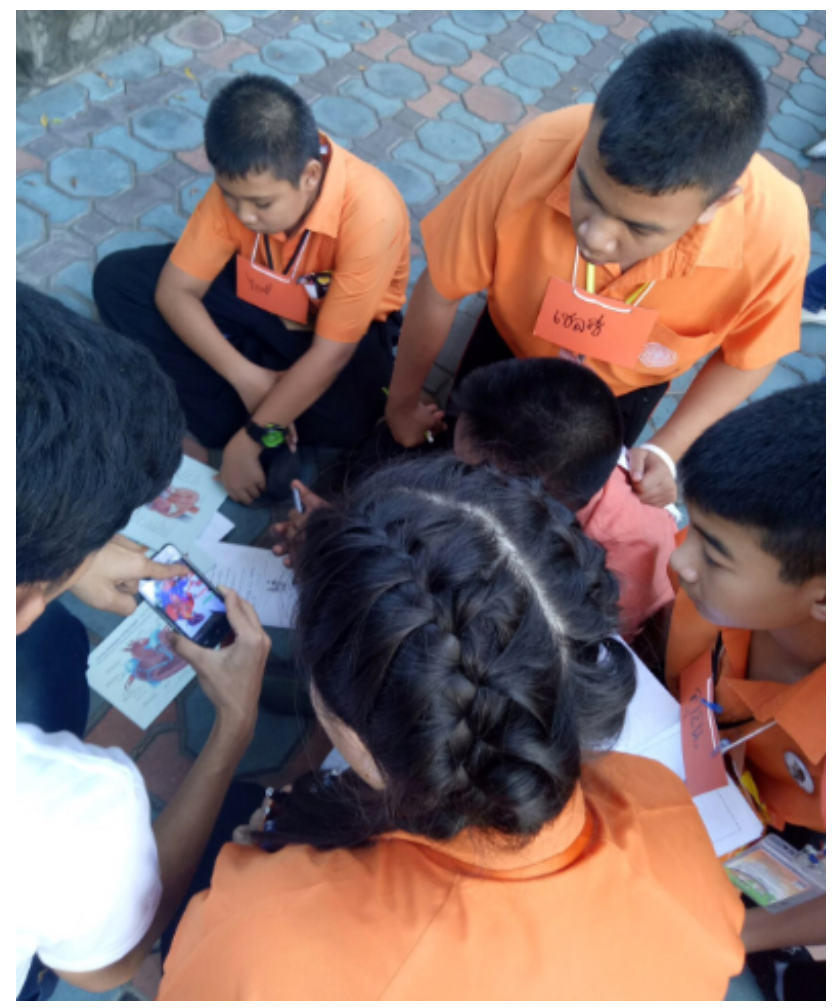

Fig. 4. Workshop demonstrating how to use the augmented reality tool to teach about the heart

\subsection{The Learning Results of the Sample Group after Learning with the Augmented Reality Tool to Teach about the Heart}

The research had developed the pre-test and post-test to obtain learning results. The tests had passed the Content Validity Index (CVI), evaluated by the five experts [15]. From the assessment of the Content Validity Index evaluated by all the experts, it was determined that the CVI value was 0.83 as there were ten questions out of twelve questions receiving the scores at 3 or 4 . As the result was higher than 0.8 (the highest CVI value was 1), the ten questions had passed the CVI. Before being trained, the sample group had to finish the ten questions pre-test. Subsequently, they would enter the training session with the augmented reality tool. It took three hours. After the training, the subjects did the post-test which had the same questions as the pretest, but the order of questions and the answers were changed. After collecting the post-test, the trainers provided the subjects with the correct answers. Completed tests were assessed, scores recorded, and data analysis was performed by using the T-Test to find comparisons between the pre-test and post-test learning results. 
2.7 Evaluating the Effectiveness of the Augmented Reality for Teaching Thai Students about the Human Heart Based on the Unified Theory of Acceptance and Use of Technology (UTAUT)

To evaluate the effectiveness of the augmented reality for teaching Thai students about the human heart based on the UTAUT [14], the research developed a questionnaire on the acceptance of the augmented reality tool to teach the sample group about the heart and evaluate the four aspects of the tool which are 1) anticipation on performance, 2) anticipation on effort, 3) social influence, and 4) the condition of facilities during the application. There were two questions for each aspect in the test. Subsequently, the data was analyzed to find the mean and the standard deviation values to determine the assessment effectiveness on the acceptance of the augmented reality tool.

\section{Results}

The results of the development of the augmented reality to teach Thai students about the human heart, include the following.

\subsection{The Test Results of the Augmented Reality for Teaching Thai Students about the Human Heart}

The pre-test and post-test results after the augmented reality training from thirty subjects revealed that the post-test results of the sample group after training was better than their pre-test results. The results were analyzed by comparing the number of the subjects who could answer the questions correctly with their individual results. The learning results showed that the subjects had more correct answers after the training. From the comparative test results collected before and after the training, according to the statistical test of the following hypothesis:

The hypothesis was assumed as followed:

H0: The learning result before and after using the augmented reality tool were not different.

H1: The learning result before and after using the augmented reality tool was different.

Statistically tested by T-Test, the main hypothesis $(\mathrm{H} 0)$ was rejected because the significance value was lower than the significance level $(\alpha)$ which was previously determined. In this study in which $\alpha=0.05$, the $\mathrm{H} 0$ was rejected and the H1 was accepted. The efficiency of the developed model was different in their methods. From the Table of Pair Sample Testing, the significance value was analyzed to consider whether the mean values of the two groups were different. In fact, it was found that the significance value was lower than the predetermined significance level. Therefore, the mean values of the two groups were different. Considering the comparative differences of the learning results both before and after the use of the augmented reality, it was found that there were differences at the statistical significance $p$ value $<0.001$. 


\subsection{The Evaluation Results of the Acceptance and the Use of the Augmented Reality for Teaching Thai Students about the Human Heart}

The results of the acceptance and the use of the augmented reality were evaluated in four aspects which are: 1) the anticipation of the performance; its arithmetic mean was 4.65 and the standard deviation was 0.48 . It showed that the acceptance of the performance was rated at the highest level; 2) the anticipation of the effort; the arithmetic mean was 4.63 and the standard deviation was 0.49 . It showed the anticipation of the effort was rated at the highest level; 3) the arithmetic mean and the standard deviation of the social influence were 4.67 and 0.48 , respectively. It showed that the social influence was accepted at the highest level. 4) The arithmetic mean and the standard deviation of the condition of facilities for the application were 4.63 and 0.49 , respectively. So, the acceptance of facilities in application was rated at the highest level. The overall image of the evaluation effectiveness results on the acceptance of the users in regard to the augmented reality tool had the arithmetic mean value of 4.65 , and its standard deviation was 0.48 . It showed that the users accepted the augmented reality as a tool to teach about the heart at the highest level as shown in Table 1 .

Table 1. The Evaluation Results of the Acceptance and the Use of the Augmented Reality for Teaching Thai Students about the Human Heart

\begin{tabular}{|l|c|c|l|}
\hline \multicolumn{1}{|c|}{ Items } & Mean & SD & \multicolumn{1}{c|}{ Decision } \\
\hline 1. The anticipation of the performance & 4.65 & 0.48 & The highest level \\
\hline 2. The anticipation of the effort & 4.63 & 0.49 & The highest level \\
\hline 3. The social influence & 4.67 & 0.48 & The highest level \\
\hline 4. The acceptance of facilities in application & 4.63 & 0.49 & The highest level \\
\hline \multicolumn{1}{|c|}{ Total } & 4.65 & 0.48 & The highest level \\
\hline
\end{tabular}

\section{Conclusion}

This paper presents the development of the augmented reality tool to teach about the human heart in a bilingual setting (Thai and English language). It was evaluated by the five experts who had the experiences in teaching and researching about the virtual biological technology using the content consistency analysis (Index of Item Objective Congruence: IOC). The IOC value of each content was 0.8 . The results indicated that the constructed AR was consistent with the objective and correspondent with the anatomy of the heart and evaluated by using the Diffusion of Innovation theory (DOI) in order to find out whether this innovation can be adopted by the sampling group. The assessment results were the arithmetic mean valued at 4.04 while the standard deviation was 0.35 . This shows that the augmented reality as a tool to teach about the heart was accepted for the dissemination at a high level. The system was tested with a group of 30 subjects who learned from the system and an experiment with a pre-test was conducted. The results of the study showed that the subjects who used the augmented reality got better results for learning. Correspondingly, there are 
four aspects of the effectiveness evaluation focusing on the acceptance of the augmented reality as a tool to teach about the heart measured by the theory of Unified Theory Acceptance and Use of Technology (UTAUT). The overall image of the effectiveness assessment results on the acceptance of the users was rated at the highest level as it the arithmetic mean and the standard deviation were 4.65 and 0.48 , respectively. To conclude, the development of the augmented reality as a tool to teach about the human heart can contribute to the effective learning and better results in understanding.

\section{$5 \quad$ References}

[1] Kaufmann, H. (2013). Collaborative Augmented Reality in Education. Instituted of Software Technology and Interactive Systems. Vienna University of Technology.

[2] Jirapan, K. (2012). Promoting Thai Cultural Art with Virtual World Technology: the Case Study of Thailand Planet Project. School Of Digital Media. Sripatum University.

[3] Jiravarapong, B. (2010). Augmented Reality. Journal of Education Naresuan University, 12(3), 1-6.

[4] Tieranabunjong, Y. (2001). Knowledge of multimedia for education. Bangkok. Kurusapa Press.

[5] Volkan, I., Bradford, W., Ruzena, B. (2007). Building a 3D Virtual Museum of Native American Basket. Proceedings Third International Symposium on 3D Data Processing, Visualization, and Transmission, 954-961.

[6] Barsom E., Graafland, M., Schijven, MP. (2016). Systematic review on the effectiveness of augmented reality applications in medical training. Surgical Endoscopy Journal, 30(10), 4174-4183. https://doi.org/10.1007/s00464-016-4800-6

[7] Wee, S. K., Benjamin, B., Kavit A., Adrian C., Ketan P., Jason, W., (2016). Augmented and virtual reality in surgery the digital surgical environment: applications, limitations and legal pitfalls. Annals of Translational Medicine Journal, 4(23), 454. https://doi.org/10.21037/atm.2016.12.23

[8] Joyce, A. C., Martin, E. T., Marie, W., Helen, R., Madeleine, K., Claes, F. L., Arianit, K., (2017). A design study investigating augmented reality and photograph annotation in a digitalized grossing workstation. Journal of Pathology informatics, 8(31).

[9] Noonchu, S. (2016). Heart Process. http://fat.surin.rmuti.ac.th/teacher/songchai/circula tory/blood circulation.htm.

[10] Turner, R, C., Carlson, L. (2002). Index of Item Objective Congruence for Multiple Objective Measures. Unpublished manuscript, University of Arkansas.

[11] Roger, E. (1995). Diffusion of Innovations. Free Press. New York.

[12] Agarwal, R.., Prasad. J. (1997). The role of innovation characteristics and perceived voluntariness in the acceptance of information technologies. Decision Science, 28(3), 557-582. https://doi.org/10.1111/j.1540-5915.1997.tb01322.x

[13] Nuanmeesri, S., Kadmateekarun P., Poomhiran, L. (2016). Publicizing Thai Dress of Royal Endorsement by Augmented Reality. The Social Sciences, 11(14), 3563-3568.

[14] Chaveesuk, S., Jaturapa, S. (2012). Theory of acceptance and use of technology. KMITL Information Technology Journal, 1(1), 1-21.

[15] Polit, D., Beck, C. (2008). Nursing research: Generating and assign evidence for nursing practice. Philadelphia, Lippincott. 
[16] Dreamstime. (2016). The pathway of blood flow through the heart. https://www.dreamstime.com/stock-image-pathway-blood-flow-heart-image19337291

\section{Acknowledgment}

I would like to express our gratitude to the Office of the Higher Education Commission for funding this research study and also Institute for Research and Development, Suan Sunandha Rajabhat University, who offered us the opportunities to conduct this research

\section{Authors}

Sumitra Nuanmeesri is with Suan Sunandha Rajabhat University, Bangkok 10130 Thailand.

Article submitted 27 February 2018. Final acceptance 25 April 2018. Final version published as submitted by the author. 